\title{
Imaging malaria sporozoites in the dermis of the mammalian host
}

\author{
Rogerio Amino ${ }^{1,3}$, Sabine Thiberge ${ }^{1}$, Samantha Blazquez ${ }^{1}$, Patricia Baldacci ${ }^{1}$, Olivier Renaud ${ }^{2}$, Spencer Shorte ${ }^{2}$ \\ \& Robert Ménard ${ }^{1}$
}

${ }^{1}$ Unité de Biologie et Génétique du Paludisme, Institut Pasteur, 25-28 rue du Dr Roux, 75724 Paris cedex 15, Paris, France. ${ }^{2}$ Plate-forme d'Imagerie Dynamique, Institut Pasteur, 25-28 rue du Dr Roux, 75724 Paris cedex 15, Paris, France. ${ }^{3}$ Present address: Departamento de Bioquimica, Universidade Federal de Sao Paulo, Rua Três de Maio 100, 04044-020, Sao Paulo, S.P., Brazil. Correspondence should be addressed to R.A. (roti@ecb.epm.br) or R.M. (rmenard@pasteur.fr).

Published online 5 July 2007; doi:10.1038/nprot.2007.120

The initial phase of malaria infection is the pre-erythrocytic phase, which begins when parasites are injected by the mosquito into the dermis and ends when parasites are released from hepatocytes into the blood. We present here a protocol for the in vivo imaging of GFP-expressing sporozoites in the dermis of rodents, using the combination of a high-speed spinning-disk confocal microscope and a high-speed charge-coupled device (CCD) camera permitting rapid in vivo acquisitions. The steps of this protocol indicate how to infect mice through the bite of infected Anopheles stephensi mosquitoes, record the sporozoites' fate in the mouse ear and to present the data as maximum-fluorescence-intensity projections, time-lapse representations and movie clips. This protocol permits investigating the various aspects of sporozoite behavior in a quantitative manner, such as motility in the matrix, cell traversal, crossing the endothelial barrier of both blood and lymphatic vessels and intravascular gliding. Applied to genetically modified parasites and/or mice, these imaging techniques should be useful for studying the cellular and molecular bases of Plasmodium sporozoite infection in vivo.

\section{INTRODUCTION}

The notion that Anopheles mosquitoes inject Plasmodium sporozoites into the dermis of the host, rather than directly into the blood circulation, was first suggested in the $1930 \mathrm{~s}^{1}$ and has since received experimental confirmation ${ }^{2-4}$. Sporozoites are ejected when the mosquito salivates ${ }^{5}$, especially when it probes the dermis, searching for a blood source. However, given the small number of sporozoites injected through its bite and the highly motile behavior of these sporozoites, the dermal phase of sporozoite infection is still poorly characterized. Only now, with the development of tools for studying the fast dynamics of sporozoites in real time, can their exact in vivo fate be analyzed.

A fundamental feature of the malarial sporozoite is its motility and migratory behavior. Like other apicomplexan parasites, this needle-shaped cell ( $10 \mu \mathrm{m}$ in length, $1 \mu \mathrm{m}$ in width) moves by gliding over a substrate at very high speeds (up to $4 \mu \mathrm{m} \mathrm{s}^{-1}$ ). The sporozoite and its gliding properties have mainly been studied in species of Plasmodium that infect rodents (P. berghei and P. yoelii), which offer, over the human-infecting parasite species, the advantages of safety in handling the parasites and ease in manipulating their genome. The rodent systems offer an additional key advantage, the possibility of studying parasites in as near as possible to their natural environment. A number of wild-type fluorescent sporozoites expressing a fluorescent marker (GFP or RFP) through a variety of stage-specific or constitutive promoters are now available in both $P$. berghei $i^{6,7}$ and $P$. yoelii ${ }^{8}$, which can now be imaged in the dermis or liver of rodent hosts ${ }^{9-12}$.

Imaging techniques have been revolutionized by advancements in both microscope instrumentation and data collection/processing software. Although one-photon methods are more limited than two-photon methods in the depth at which they can image, the one-photon confocal microscope can nonetheless collect data at depths of up to $80 \mu \mathrm{m}$ from the tissue surface, and is highly effective in the skin. Although traditional confocal systems rely on slow laser scanning of the sample, which limits their capacity to record fast cellular processes, the use of a spinning-disk confocal microscope, which excites many points simultaneously, can overcome this limitation by collecting data faster ${ }^{13}$. A confocal microscope captures $x-y$ planar signals from fluorescent targets, which are combined into volume information by sequentially capturing successive $z$-plane images. Repeating the collection of $3 \mathrm{D}$ data produces a $4 \mathrm{D}$ data set (3D volume plus time) that can be used to create a movie clip presenting an accelerated view of the dynamics of the fluorescent source.

We present here a protocol for sequentially imaging fluorescent sporozoites and the blood circulation in a defined volume of mouse skin. The rapid motions of the parasites and the blood flow are recorded in real time using a high-speed spinning-disk confocal microscope. Using a $\times 10$ objective, the camera field covers an area of approximately $870 \times 660 \mu \mathrm{m}^{2}$. This surface is sufficient to follow the behavior of most of the parasites present in a static microscope field and thus to perform quantitative studies, because only a small proportion of the parasites that are initially visible leave (by gliding) the field of observation during 1-h recordings. To gather as much information as possible, five $\mathrm{z}$-slices ( $15 \mu \mathrm{m}$ each) are recorded to yield an observed volume of approximately $870 \times$ $660 \times 60 \mu \mathrm{m}^{3}$. Each time point thus consists of five $\mathrm{z}$-slices in the parasite channel and five $\mathrm{Z}$-slices in the blood channel, totalizing two Z-stacks per time point. As the parasite moves in the dermis at velocities close to $2 \mu \mathrm{m} \mathrm{s}{ }^{-1}$, the maximum interval between two time points should ideally be $<6 \mathrm{~s}$ to avoid discontinuity in the recording of parasite displacement. This protocol permits visualization of the sporozoites' path in the dermis, parasite binding and invasion of vessel walls, as well as gliding inside vessels.

The only quantitative study on sporozoite fate in the dermis published so far $^{11}$ has reported unexpected findings. Sporozoites 
appear to have distinct fates in the dermis: they can invade blood vessels and eventually reach the liver, invade lymphatic vessels and accumulate in the draining lymph node, but can also remain in the dermis. The important host-parasite interactions involved in the 'skin phase' of the parasite's life cycle must now be characterized. By capitalizing on the large collection of available knockout mice or mice displaying cell-lineage-specific fluorescence, as well as on the growing number of sporozoite mutants with distinct defects, imaging promises to be highly informative at the tissular, cellular and molecular levels.

\section{MATERIALS}

\section{REAGENTS}

-18-22-d post-infection Anopheles stephensi Sda500 mosquitoes (containing 10,000-30,000 P. berghei sporozoites per salivary gland $)^{14}$

- P. berghei NK65 clone, expressing GFP under the control of the csp promoter (stage-specific fluorescence at the oocyst, sporozoite and early liver stages $)^{6}$

- P. berghei ANKA clone, $\mathrm{PbGFP}_{\mathrm{CON}}$, expressing GFP under the control of the ef $-1 \alpha$ promoter (the parasite is fluorescent at all stages) ${ }^{7}$

- Mice: 4-7-week-old hairless SKH1 females (Charles River Laboratories) I CAUTION All experiments involving rodents must conform to National and Institutional regulations. This protocol was approved by the committee of the Pasteur Institute.

-1-ml insulin syringes (Terumo, cat. no. BS-01T)

- Needle $30 \mathrm{G} \times 0.5$ in. (BD Microlance, cat. no. 304000)

- Anesthetic solution comprising Imalgene 1000 (Ketamine; Merial) and Rompun 2\% (Xylazine; Bayer), or solely Pentobarbital (Ceva, France) (see REAGENTS SETUP)

- PBS Dulbecco 1X (Invitrogen, cat. no. 14040091)

- BSA conjugated to Alexa Fluor 647 at $1 \mathrm{mg} \mathrm{ml}^{-1}$ (Molecular Probes-Invitrogen)

- Coverslips (micro cover glass $24 \mathrm{~mm} \times 60 \mathrm{~mm}$; Eric Scientific Company)

-3M Scotch tape

EQUIPMENT

- Aspirator for collecting mosquitoes

- Stereozoom microscope with epi-fluorescence and GFP filter for separating mosquitoes (Zeiss Stemi 2000-C; Carl Zeiss) with fiber-optic lighting (Schott KL1500LCD)

- Net-covered cage for infected mosquitoes

- High-speed spinning-disk confocal system (UltraVIEW ERS; Perkin Elmer, http://las.perkinelmer.com/content/livecellimaging/) mounted on an inverted Axiovert 200 microscope (Carl Zeiss) (see EQUIPMENT SETUP)

- High-speed Orca ER camera (Hamamatsu, Japan). Note that this camera is sufficient for the acquisitions presented; however, faster cameras are commercially available.

- $\times 10$ Plan-NeoFluar NA 0.3 or a $\times 25$ Plan-NeoFluar NA 0.8 multiimmersion corrected objectives for the spinning-disk system (Carl Zeiss)

- Homeothermic blanket system for animal heating (Harvard Apparatus) http://www.harvardapparatus.com/webapp/wcs/stores/servlet/

category_11051_10001_37612_-1_HAI_Categories_N_37611_37610
- Hand-made aluminum platform or rectangular Petri dish for cell culture to hold the mouse on the motorized microscope stage (see EQUIPMENT SETUP)

- Perkin Elmer UltraVIEW ERS Image Suite software (image acquisition) - ImageJ (image analysis) http://rsb.info.nih.gov/ij; click on the 'Download' option and install 'ImageJ'. Click on 'Plugins > Stacks', and install 'MultiTracker Plugin' (http://rsb.info.nih.gov/ij/plugins/multitracker.html) REAGENT SETUP

Ketamine/xylazine anesthetic solution Mix 2 volumes of Imalgene 1000 (ketamine) with 1 volume of Rompun 2\% (xylazine) and 5 volumes of PBS $1 \times$. For mice, inject i.p. $\sim 50 \mu \mathrm{l}$ of this solution for each $10 \mathrm{~g}$ mouse body weight (ketamine: $125 \mathrm{mg}$ per kg body weight, xylazine: $12.5 \mathrm{mg}$ per $\mathrm{kg}$ body weight). Store the solution at $4^{\circ} \mathrm{C}$.

Pentobarbital anesthetic solution Dilute pentobarbital in PBS $1 \times$ to obtain a solution at $50 \mathrm{mg}$ per kg body weight, inject i.p. $\sim 50 \mu \mathrm{l}$ of this solution for each $10 \mathrm{~g}$ of mouse body weight.

\section{EQUIPMENT SETUP}

Live cell imaging using a high-speed spinning-disk confocal system Our fluorescent imaging was performed using a high-speed spinning-disk confocal system equipped for triple-wavelength excitation ( $\mathrm{Kr}-\mathrm{Ar}$ laser; 488,568 and $647 \mathrm{~nm}$ ) controlled through an acousto-optical tunable filter (AOTF) device. Axial through-stack or 'Z-stack' sampling ${ }^{15}$ used an objective piezo-drive system (Physik Instrumente, Germany), controlled and synchronized to the imaging system using the Perkin Elmer pilot software. The imaging system was built on a Zeiss Axiovert 200 manual microscope equipped with a $\times 10$ Plan-NeoFluar objective and a $\times 25$ Plan-NeoFluar multi-immersion corrected objective. Fluorescent images were recorded using a Hamamatsu (Japan) Orca ER camera and used exposure times in the range $200 \mathrm{~ms}-1 \mathrm{~s}$. Fast axial (through-stack) sampling used a piezo-objective drive stepping at $10-15 \mu \mathrm{m}$ along the $z$-axis or $0.5 \mu \mathrm{m}$, depending on the experiment.

Microscope platform The platform we used to hold the animal on the microscope stage is a handmade aluminum adapter $(160 \mathrm{~mm} \times 110 \mathrm{~mm}$ $\times 2 \mathrm{~mm}$, the same measurements as the microscope stage opening) with a round central opening of diameter $21 \mathrm{~mm}$. A coverslip is placed on the central hole of the platform and fixed by taping the extremities onto the adapter. If it is not possible to obtain the aluminum adaptor, the cover of a rectangular Petri dish for cell culture can be used, by making a hole in the center of the cover. The hole must be slightly smaller than the size of the coverslip that is used.

\section{PROCEDURE}

\section{Selection of mosquitoes with infected salivary glands}

1) $18-22 \mathrm{~d}$ before the imaging session, infect mosquitoes as described in ref. 14 .

2| At least $24 \mathrm{~h}$ before the bite session (Steps 9-11), separate infected from noninfected mosquitoes (18-22 $\mathrm{d}$ after mosquito infection, when sporozoites have maximal infectivity). Using an aspirator tube, collect female mosquitoes, anesthetize/immobilize the collected insects by blowing them into a 15-ml Falcon tube on ice and carefully transfer the cold-anesthetized insects onto a Petri dish placed on ice.

3| Examine the mosquitoes under a stereomicroscope with epi-fluorescence (GFP filter), remove the mosquitoes that do not display GFP fluorescence in the salivary glands (Fig. 1) and delicately transfer fluorescent mosquitoes to a net-covered cage. $\triangle$ CRITICAL STEP As all mosquitoes in a cage are usually not infected, and given that imaging is performed after a single mosquito bite, it is crucial to select mosquitoes with infected salivary glands for the bite session. Select at least 20 mosquitoes with fluorescent salivary glands. Keep these mosquitoes at $21^{\circ} \mathrm{C}$ and at least $70 \%$ humidity, but without sugar solution to starve the mosquitoes and increase their biting behavior the next day. 


\section{Preparing for the imaging session}

4| Prepare the recipient rodent(s) and the selected mosquitoes. Ideally, imaging is performed using hairless mice to avoid autofluorescence of the hair, but $\mathrm{C57BL} / 6$ and BALB/C mice, as well as Brown-Norway rats, have been used successfully after shaving of the ear pinna.

5| Turn on the homeothermic heater blanket, install the desired objective $(\times 10$ or $\times 25)$, turn on the imaging system and verify that all electronic devices of the imaging system are working properly (lasers on, fan working, motorized stage moving, mercury arc lamp on, software running).

6| In the microscope software, open the windows necessary for image acquisition. For tracking sporozoite movement, it is important to privilege rapid acquisition using a large $z$-slice (approximately $10 \mu \mathrm{m}$ ). For studying sporozoite-host cell interaction, it is important to privilege small $z$-slice intervals, with or without time. To reduce enormous data files, a binning of $2 \times 2$ allows sufficient information for cell tracking and cell interactions. The binning can be adjusted according to the desired temporal-spatial resolution. The smaller the binning (e.g., $1 \times 1)$, the better the $x, y$ spatial resolution; however, temporal resolution will be worse (longer exposure times needed) and the files will be bigger. In Image Suite, open the following windows: 'Gallery', 'Experiment', 'Image Control', 'Hardware Controller' and 'Sequence Control'. In 'Gallery', open a new experiment. Go to the 'Image Control' window, set the binning to $2 \times 2$, increase the gain to the maximum (255) and click on 'full chip' button. Go to the 'Hardware Control' window, 'Dichroic' panel, click on the '488/568/647' button. In the 'Channel settings' panel, channel 'A' option, select the laser to excite on $488 \mathrm{~nm}$ and set it to record. In channel ' $B$ ', select the laser to excite on $647 \mathrm{~nm}$ and set it to record. Adjust the 'Exposure' time to $500 \mathrm{~ms}$. In the 'Power' panel, adjust the laser for maximum power. In 'Stage Control', set the slice spacing to $10 \mu \mathrm{m}$ if using a $\times 25$ objective, or to $15 \mu \mathrm{m}$ if using a $\times 10$ objective. For punctual Z-stacks of cell interactions, the $z$-slice should be reduced, for example, to $0.5 \mu \mathrm{m}$. To achieve an optimal 3D reconstruction, the z-step must be at most equal to the thickness of the optical slice or smaller.

$\triangle$ CRITICAL STEP The parameters stated here are only an indication and can vary depending on both the samples and the equipment used.

7| Test the laser-In the 'Hardware Controller' window, 'Channel settings' panel, click on the channel ' $A$ ' button, and a blue light should be seen; click on the channel ' $B$ ' button, and a red light should be seen.

! CAUTION The laser beam can cause serious eye damage. Note that the energy measured at the end of the objective with the spinning disk confocal laser is one order of magnitude lower than the energy from a laser-scanning confocal and two orders of magnitude lower than the energy from the mercury arc lamp.

8| Fix a coverslip with adhesive tape over the hole in the platform (see EQUIPMENT SETUP). Install the platform on the microscope and cover with the thermal blanket.

\section{Obtaining the imaging sample}

9| Anesthetize the recipient mouse (approximately $20 \mathrm{~g}$ ) by i.p. injection of approximately $100 \mu \mathrm{l}$ anesthetic.

10| Hold the anesthetized animal gently over the cage containing the infected mosquitoes exposing the periphery of the ear pinna (external face) to the selected mosquitoes. Wait for a mosquito to land on the ear and let the insect bite for 1 min. Note the time of bite by using the time on the computer, so that this will correspond to the times on the image files. For example, double-click on the clock at the bottom right corner of the computer to monitor the time of bite. The UltraVIEW software uses the same clock to save the time in the recorded files.

$\triangle$ CRITICAL STEP This step requires patience; it can take from a few minutes to hours. In general, $40 \%$ of $A$. stephensi mosquitoes have found a blood source on which to feed in the minute that follows landing on the prey ${ }^{16}$. Studies on the sporozoite ejection rate during mosquito salivation ${ }^{5,17}$ have revealed that mosquitoes harboring thousands of sporozoites in their salivary glands could eject either no or over 1,000 sporozoites during a 10-min-long salivation period, while during the first minute of salivation, a mean of 
20 sporozoites is ejected. Overall, although the ear pinna is ideal for imaging because of its accessibility and thinness, other sites can be imaged. Note that bites can provoke a local hematoma or not; in the latter case, the site of bite should be memorized using the pattern of superficial veins and marked using nonfluorescent ink.

\section{? TROUBLESHOOTING}

11 Remove the thermal blanket from the microscope stage, place the animal on the heated platform and accommodate the hematoma/bite site at the center of the coverslip, taped on the hole of the platform. Then, tape the ear pinna as flatly as possible to the coverslip.

12| Switch on the mercury arc lamp, turn the light path toward the ocular and search for sporozoites using a GFP/ fluorescein isothiocyanate filter. If no sporozoites are seen, repeat Steps 11 and 12 .

$\triangle$ CRITICAL STEP Avoid lengthy exposure to mercury arc lamp illumination; it can cause excess heating of the sample or cell damage via the generation of oxygen radicals and other forms of photoxicity. Many of the deleterious effects are cumulative, which limits the time in which reliable observations can be made.

13| Remove the platform with the animal from the microscope carefully, inject $50 \mu \mathrm{l}$ fluorescent BSA into the retro-orbital sinus using an insulin syringe to label the blood circulation and, if the animal starts to wake up, inject i.p. $25 \mu \mathrm{l}$ anesthetic.

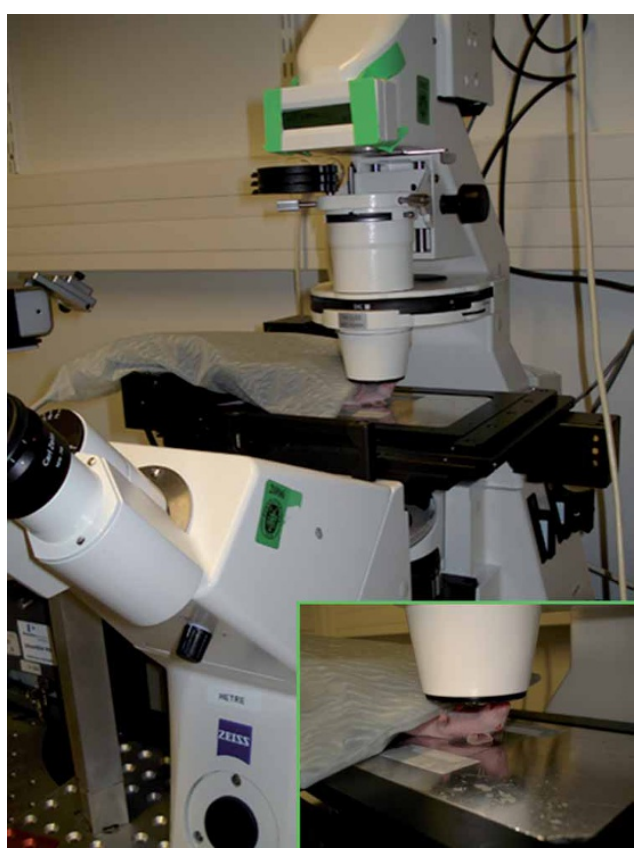

Figure 2 | Experimental setup for imaging sporozoites in the dermis of the murine ear. Once the mouse has been bitten, it is placed on the microscope platform so that the site of bite (on the ear) is in the middle of the coverslip over the hole. The ear is then taped into place to avoid any displacement during recording, and the mouse is covered with the homeothermic blanket.

14| Place the platform back on the microscope stage, cover the animal with the thermal blanket, making sure that the bite site is at the center of the hole and find the sporozoites using the mercury arc lamp (Fig. 2).

15| Prepare for image acquisition by changing the excitation light path from the mercury arc to the laser source and the emission light path from the ocular to the detector. Other changes may be necessary, such as the position of microscope filters. ? TROUBLESHOOTING

\section{Acquiring the images}

16| Activate the 'live image' window and the 488-nm laser to visualize GFP-expressing parasites. Use the microscope controls (focus adjustment knobs and the motorized microscope stage) to focus on the parasites and define the observation field. If there are many parasites in the same field, numerous events will be recorded at the same time. Sporozoites move rapidly, so try to avoid parasites at the edges, as they may move out of the field during the recording. In the 'Experiment' window, click on the 'Live Images' button. Go to the 'Hardware Controller' window in the 'Channel settings' option, and click on the channel $A$ and activate the excitation on $488 \mathrm{~nm}$. The camera image should appear on the monitor.

? TROUBLESHOOTING

\section{Focusing}

17| Focus on the epidermis using the fine adjustment knob and set this as the bottom z-position. In the 'Hardware Controller' window, 'Move' panel, position the z-step controller on $80 \mu \mathrm{m}$. The depth of the focal plane is now controlled using the z-step controller in the software.

\section{? TROUBLESHOOTING}

\section{Setting Z-stacks ('3D snapshots')}

18| Using the $z$-step controller (positioned at the bottom, i.e., $80 \mu \mathrm{m}$ ), move the focus toward the top position (i.e., $0 \mu \mathrm{m}$ ). When the first (most superficial) parasite appears, set the Z-stack bottom limit. Using the $z$-step controller, continue moving toward the $0 \mu \mathrm{m}$ position. When the deepest parasite is out of focus, set the top limit of the Z-stack.

$\triangle$ CRITICAL STEP Usually, a Z-stack of $50 \mu \mathrm{m}$ is enough to cover the skin volume that contains the parasites delivered during a bite. If the parasite dispersion exceeds $50 \mu \mathrm{m}$, using the microscope, focus on the most superficial parasite and reset at that $z$-position the Z-stack bottom limit (i.e., $80 \mu \mathrm{m}$ ). This step should be done as fast as possible, and is just one among the several ways for adjusting the Z-stack settings.

? TROUBLESHOOTING 


\section{Setting exposure time and laser power}

19| Adjust the exposure time and laser power for each laser (i.e., on channel ' $A$ ' (488 nm) and 'B' (647 nm)), so that the blood vessels and the parasites are visible.

$\triangle$ CRITICAL STEP The time of exposure should be adjusted depending on the number of slices per channel. If the laser power is not already on maximum, it is possible to add power, to keep the exposure time as low as possible. To minimize photobleaching, keep the laser power and total exposure time as low as possible. For time lapse, the ideal conditions are determined by the duration of the experiment with a minimum acquisition time, and a minimum sampling frequency for Z-stacks (i.e., the longest time possible between each Z-stack acquisition, which still allows reasonable temporal resolution of the parasite dynamics; in our case, there is no delay between stacks). In contrast, each Z-stack acquisition should be achieved with the minimal delay between images (ideally continuous acquisition) and the shortest exposure time per image.

? TROUBLESHOOTING

\section{Recording movies}

20| Set the system to record sequences of $10 \mathrm{~min}$. In the 'Sequence Control' window, go to 'Length (sec)' option and set the recording time (we usually record movies of $600 \mathrm{~s}$; see Step 28). Go to 'Grab Options', activate the Z-stack (green) and the time-lapse (red) buttons. Start recording the images by clicking on the gray button with a red circle at the bottom of the 'Sequence Control' window. The 'Experiment' window should show the real-time acquisition.

$\triangle$ CRITICAL STEP Looking at the real-time images, check if all parameters (laser, $z$-step and time of exposure) are properly set. If not, stop the acquisition, correct the parameter and restart recording; i.e., if parasites are not visible, verify that the 'green' channel is being recorded and that the time of exposure is correct.

21| When the time of acquisition is completed, check the quality of the recorded images. For example, if parasites remain visible and their movement can be followed, then more sequences can be recorded until the desired length of time has been acquired. In the 'Sequence Control' window, click (gray button with red circle) to record again. Note that when using the Hamamatsu camera (full chip), each image (binning $2 \times 2$ ) has $672 \times 512$ pixels (approximately $340 \mathrm{~kb}$ ). When recording $600 \mathrm{~s}$-long movies, recording $5 \mathrm{z}$-slices in both the green and far-red channels with an exposure time of $400 \mathrm{~ms}$ per time-point, the computer generates 1,500 pictures to $500 \mathrm{Mb}$ per film.

$\triangle$ CRITICAL STEP If necessary, more anesthetic $(25 \mu \mathrm{l})$ can be injected during recording, but do not move the animal.

\section{Exporting the images}

22| Export the images as .tif files using the UltraVIEW software. Alternatively, use the Perkin-Elmer 4D-images series converter plugin, written to run in ImageJ, which is freely available at http://www.embl-heidelberg.de/eamnet/html/pe_plugin.html (Marchand, M. and Shorte, S.L., PFID, Institut Pasteur).

PAUSE POINT Once the images have been saved, they can be analyzed immediately or at a later point.

\section{Processing the images: ImageJ and plugins}

23| Open exported images: in 'File > Import > Image Sequence', select the folder that contains the .tif files exported using the UltraVIEW software. In 'Sequence option > File name contains', type the file name (e.g., ' $488 \mathrm{~nm} \mathrm{Z0').} \mathrm{The} \mathrm{software} \mathrm{will}$ open all files containing this name or all the time points of Z-stack 0 acquired on the 488-nm channel.

\section{Visualizing the position of sporozoites in the Z-stack over time}

24| The output of imaging is often presented as movie clips made by projecting the $z$ information into a single image by using a method called maximum-intensity projection. This process removes information on where the cells actually lie in the z-dimension, which may result in the impression that two cells come into contact when they actually pass over each other. One method to provide more $z$-dimension information for the viewer is to change the color of the target dynamically, based on its $z$-position within the image volume. For instance, the $z$-slice from 0 to $10 \mu \mathrm{m}$ in blue, the $z$-slice from 11 to $20 \mu \mathrm{m}$ in green and so forth.

\section{Visualizing sporozoite movement over time: merging Z-stacks over time}

25| To merge three time sets of Z-stacks (ZO, Z1 and Z2), as shown in Figure $\mathbf{3}$ (see panel $\mathbf{b}$ ), open each TZ-plane (time points of a given $z$-plane) as one stack (time points of $Z_{488} \mathrm{~nm}=$ stack0, time points of $Z 1_{488 \mathrm{~nm}}=$ stack 1 and time points of $\mathrm{Z}_{488 \mathrm{~nm}}=$ stack2). In 'Process > Image Calculator', select the stack0 as 'Image1', the stack1 as 'Image2' and the operation as 'Max' (maximum-intensity projection). Click on 'Create New Window' and on the OK button. The resulting stack (stack3) corresponds to the maximum-intensity projections of stack0 and stack1. Repeat this step, now using stack3 as 'Image1' and stack2 as 'Image2'. The resulting stack (stack4) is the maximum-intensity projection of Z0, Z1 and Z2 over time points.

26| To project the time points of stack4, go to 'Images > Stacks > Z-projection' and choose first and last time-points desired for the projection by typing the respective slice number in the options 'start' and 'stop'. Choose the way the slices between 'start' and 'stop' are projected. 
Figure 3 | Example of a parasite invading a lymphatic vessel (see Supplementary Video 3 ). (a) Experimental design. Each time point (Tp) comprises six z-slices recorded sequentially using the laser excitation at $488 \mathrm{~nm}$ and six z-slices using the excitation at $647 \mathrm{~nm}$. The exposure time of a single $z$-slice is $200 \mathrm{~ms}$, totaling $1.2 \mathrm{~s}$ per laser channel and $2.4 \mathrm{~s}$ per Tp. The $z$-step is $10 \mu \mathrm{m}$ using a $\times 25$ immersion objective and binning $4 \times 4$. (b) Temporal color code. The picture shows the changing of a continuous movement (gliding in red) to a discontinuous passive drifting (lymph drainage in green). The picture is composed by maximum-intensity projections (MIPs) of three Z-stacks (Z-projections) and time points (T-projections), using images acquired on the 488-nm channel. The first step is a Z-projection of three Z-stacks (MIP of Z0, Z1 and Z2; volume of $20 \mu \mathrm{m}$ ) for each time point. These Z-projections between the time points 0 and 40 are again maximum projected (T-projection $0-40$ ). After these two steps, the resulting projection represents the movement of the parasite in a flat volume of $20 \mu \mathrm{m}$ for $96 \mathrm{~s}$ (T-projection ${ }_{0-40}$ of Z-projection $\left.{ }_{\mathrm{Z} 0, \mathrm{Z1}, \mathrm{Z2})}\right)$. The same is performed with the merged Z-stacks between the time points 41

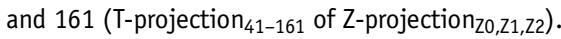
The MIP that corresponds to the gliding movement of the parasite in a volume of $20 \mu \mathrm{m}$ (T-projection O-40 $_{\text {of Z-projection }}$ Z0,Z1,Z2) was pseudo-colored in red, and the MIP of the lymph drainage (T-projection ${ }_{41-161}$ of Z-projection ${ }_{\mathrm{Z0,Z1,Z2}}$ ) was pseudo-colored in green. Scale bar $=15 \mu \mathrm{m}$. (c) Tracking the parasite. The sequence containing

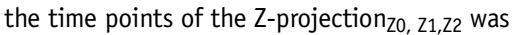
thresholded in ImageJ, and the position of each parasite was tracked using the 'multitracker' plugin. The $(x, y)$ position of the selected parasite was plotted for each time point using Microsoft Excel. The velocity is an approximate value of the average velocity between two time points and is calculated by dividing the parasite displacement $(\Delta S)$ by the time between two time points $(\Delta T=2.4 \mathrm{~s})$. Note the decrease in the velocity when the parasite changes from continuous gliding to passive drifting. (d) Color code of the $z$-planes. The picture shows that the trajectory of the passively drifting parasite does not coincide with the trajectory of the blood vessels. Each z-plane, acquired using the 488- and 647-nm channels (GFP/parasite and BSA-647/blood, respectively), was projected (MIP) over time and pseudo-colored. T-projection $0-161$ of $\mathrm{ZO}_{488}$ and $647 \mathrm{~nm}$ was pseudo-colored in blue and represents the parasite and the blood circulation at $0 \mu \mathrm{m}$. T-projection ${ }_{0-161}$ of $Z 1_{488}$ and $647 \mathrm{~nm}$ was pseudo-colored in green and represents the depth of $10 \mu \mathrm{m}$. T-projection ${ }_{0-161}$ of $Z 2_{488}$ and $647 \mathrm{~nm}$ was pseudo-colored in red and represents the depth of $20 \mu \mathrm{m}$. Note the change in color when the parasite switches from continuous gliding to passive drifting, and when the parasite crosses the vessel (white arrowhead).

\section{Tracking parasites}

27| Adjust the threshold of the merged Z-stack in 'Image > Adjust > Threshold'. Ideally, the thresholded parasite should appear as a continuous object in all slices tracked. One example is given in Supplementary Videos 1 and 2. Track the thresholded parasites by running the plugin MultiTracker in 'Plugins > Stacks > MultiTracker'. The software will generate a 'Results' table with the $(x, y)$ positions of all tracked objects.

28| Export the 'Results' table to a Microsoft Excel sheet and plot the $(x, y)$ trajectory to confirm the parasite track, as shown in Figures $\mathbf{3 b}$ and $\mathbf{4}$ (see panel b). If it is correct, calculate the distance in pixels, as shown in Figure 3c. Also see Supplementary Video 3. Convert the distance to micrometers, and divide by the time between two time points. The result is an average velocity of the thresholded parasite between two sequential images. Note that this velocity is an approximate value since the three Z-stacks are merged.

\section{Visualizing parasites and the blood circulation: pseudo-coloring of stacks}

29| A color code can be used to discriminate channels of acquisition (e.g., 488 and $647 \mathrm{~nm}$ in Fig. 4a), time points (Fig. 3a), Z-stacks (Fig. 3d), etc. ... In 'Image > Color > RGB merge...', choose the stacks and pseudo-color them in red, green and blue. 


\section{TIMING}

Step 1, infecting and rearing mosquitoes: $18-22 \mathrm{~d}$

Steps 2-3, selecting infected mosquitoes: 30 min

Steps 4-8, preparation of microscope system and anesthesia of rodent: approximately $30 \mathrm{~min}$

Steps 9-12, bite session: from minutes to hours

Steps 13-19, blood flow labeling and microscope settings: a few minutes

Steps 20, 21: time of recording: approximately $1 \mathrm{~h}$

Steps 22-29: exportation and analysis of images: largely depends on which analyses are to be done. However, the analysis steps can be done at a later time.

\section{? TROUBLESHOOTING}

Troubleshooting advice can be found in Table 1.

TABLE 1 | Troubleshooting for in vivo imaging of fluorescent parasites in the mouse dermis.

\begin{tabular}{llll}
\hline Step & Problem & Possible reason & Solution \\
\hline 10 & $\begin{array}{l}\text { Selected mosquitoes are } \\
\text { dying }\end{array}$ & Rearing conditions & $\begin{array}{l}\text { Check temperature, humidity and avoid physical stress when } \\
\text { selecting them }\end{array}$ \\
10 & Mosquitoes are not biting & Hypothermic mouse & $\begin{array}{l}\text { Do not wait too long between the anesthesia and the biting session } \\
\text { Attract the mosquitoes to the top of the cage with the heat of your } \\
\text { finger or breathing }\end{array}$
\end{tabular}
15,16 Image is not appearing in
Camera is not working
Check camera the 'Experiment' window

Laser is not working

Check laser and if channel A/B is activated

Wrong light pathway

Change the light pathway (Step 15)

Software in view mode

Activate 'Live Images' (Step 16)

17 Epidermis is not in focus

18 No parasites are visible in the 'live image' window

Poor resolution of the blood flow

Autofluorescence of the epidermis
BSA leakage from a hemorrhage

The ear has not been taped to the coverslip properly

The $z$-position on the microscope and the camera are not always exactly the same

Bad injection

The skin is autofluorescent in the green and red channels
Check that the ear is flat

Move the $z$-position slightly to see if the parasites appear on the screen

Check an ear vein for the presence of BSA-647 in the circulation. If the fluorescence is weak, reinject the BSA in the retro-orbital sinus

Use the counter side ear pinna and restart from Step 11

Blood vessels are only visible in the far-red channel, and parasites are only visible in the green channel. Before starting the acquisition, a single Z-series can be acquired using excitation at 488, 568 and $647 \mathrm{~nm}$. Color-merge these $z$-stacks as 488 (green), 568 (red) and 647 (blue). In the merged picture, the GFP signal will be green, the autofluorescence yellow or white and the BSA-647 blue

\section{ANTICIPATED RESULTS}

This protocol, which is based on the technology of spinning-disk confocal microscopy that allows for high-speed acquisition of images in a living animal without leading to tissue damage, is useful to image in 4D the behavior of fluorescent Plasmodium sporozoites in the dermis of rodents. Several crucial steps during the infectious process can now be imaged in vivo in quantitative terms, including their motility and interactions with host cells in the dermis, and their passage into the blood or the lymphatic circulation. Examples of images acquired using this protocol are shown in Figures $\mathbf{3}$ and $\mathbf{4}$. Figure $\mathbf{3}$ shows a parasite invading a lymphatic vessel (see Supplementary Video 3). The invasion event is characterized by the switching of a continuous gliding movement (red projection) to a passive drifting (green projection) (Fig. 3a). Figure $\mathbf{3 b}$ indicates the decrease of parasite velocity over time. Figure $3 \mathbf{c}$ shows the noncoincidence of the path of the passively drifting parasite with the trajectory of the blood. Figure 4 shows a parasite invading a blood vessel and gliding inside the vessel. The colored lines and squares in Figure $\mathbf{4 b}$ represent the blood flow carrying the parasite inside the vessel (see Supplementary Video 4). 
Figure 4 | Example of a parasite invading and gliding inside a blood vessel (see Supplementary Video 4). (a) Channel color code. The picture shows a parasite invading a blood vessel. The parasite (green), after invading the vessel (red and blue), glides inside the vessel and is sometimes carried by the blood flow. The image is an RGB image composition of a maximumintensity T-projection $0-91$ of one Z-stack $\left(Z_{2} 488 \mathrm{~nm}\right)$, which represents the parasite movement (in green); a maximum intensity T-projection ${ }_{0-91}$ of one Z-stack $\left(Z_{647} \mathrm{~nm}\right)$, which represents the blood movement during the time point 0-91 (in red) and an average intensity T-projection of ten selected images where the BSA-647 filled the vessel, representing the space occupied by the blood flow or the interior of the blood vessel (in blue). (b) Tracking the parasite. The graphs show the trajectory (left) and velocity (right) of the parasite invading the blood vessel. The intervals when the parasite is moving at high-speed 'jumps', concomitantly with the blood flow, are shown as $\mathrm{red} / \mathrm{green}$ lines in the trajectory graph (left) and as red/green squares in the velocity graph (right), confirming the parasite location inside the vessel. Tp0 (time point 0 ) represents the beginning of the movie. Scale bars $=15 \mu \mathrm{m}$.
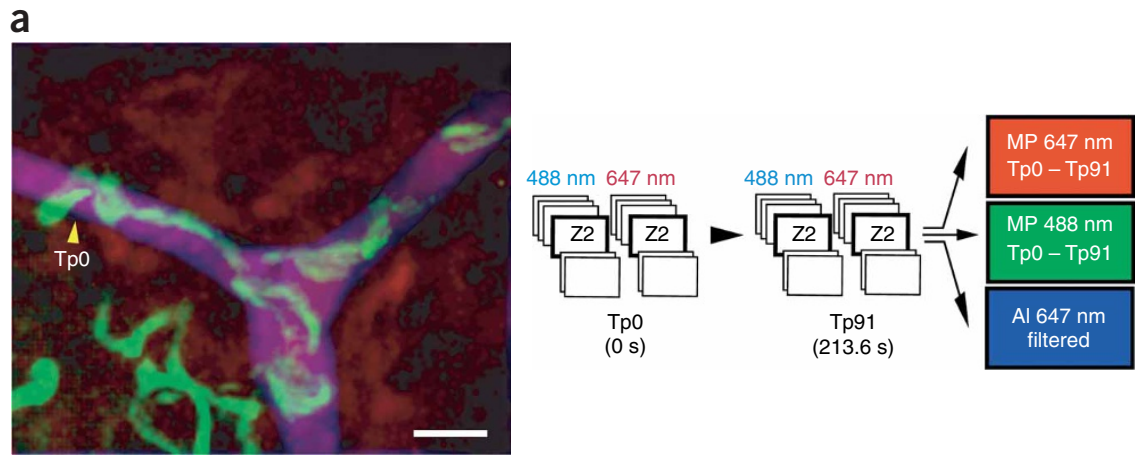

b

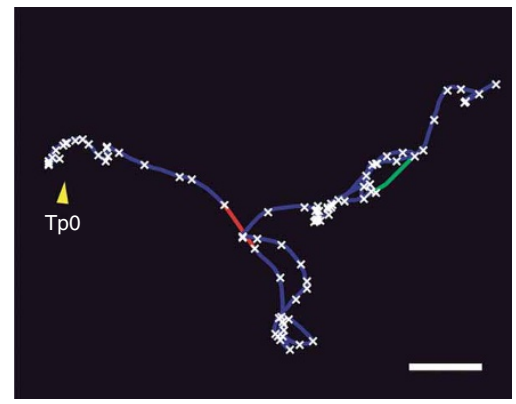

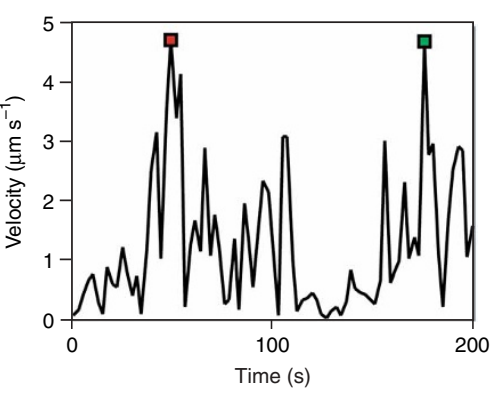

The future applications of this protocol are numerous. First, analyses using the collection of mutant parasite strains available will improve our understanding of key molecular components necessary for parasite invasion and development. Second, the use of knockout mouse strains will aid in the identification of host components involved in the fate of the parasite. For example, the study of the behavior of parasites in the dermis could be tackled using mice strains affected for extracellular matrix components or endothelial cell junction molecules. In addition, this methodology will be suitable for the study of Plasmodium sp. in their natural host (P. berghei-Grammomys surdaster) or P. falciparum in humanized mice.

Note: Supplementary information is available via the HTML version of this article.

ACKNOWLEDGMENTS We thank G. Milon, B. Martin, F. Frischknecht and P. Brey for experiment-related advice and 'real-time' support; A. Genovesico, C. Zimmer and J.C. Olivo-Marin for helping with tracking analysis; E. Perret, P. Roux, M. Marchand and C. Machu for helping with imaging; C. Bourgouin, I. Thiery and the other members of the Center for Production and Infection of Anopheles for rearing the mosquitoes and B. Franke-Fayard, A. Waters and C. Janse for providing the PbGFP CON $_{\text {parasite. This }}$ work was supported by funds from the Pasteur Institute (Stratagic project 'Grand Programme Horizontal Anopholes'), the Howard Hughes Medical Institute and the European Commission (FP6 BioMalPar Network of Excellence).

COMPETING INTERESTS STATEMENT The authors declare no competing financial interests.

Published online at http://www.natureprotocols.com

Reprints and permissions information is available online at http://npg.nature.com/ reprintsandpermissions

1. Boyd, M.F. \& Kitchen, S.F. The demonstration of sporozoites in human tissues. Am. J. Trop. Med. Hyg. 19, 27-31 (1939).

2. Ponnudurai, T., Lensen, A.H., van Gemert, G.J., Bolmer, M.G. \& Meuwissen, J.H. Feeding behaviour and sporozoite ejection by infected Anopheles stephensi. Trans. Roy. Soc. Trop. Med. Hyg. 85, 175-180 (1991).

3. Sidjanski, S. \& Vanderberg, J.P. Delayed migration of Plasmodium sporozoites from the mosquito bite site to the blood. Am. J. Trop. Med. Hyg. 57, 426-429 (1997).

4. Matsuoka, H., Yoshida, S., Hirai, M. \& Ishii, A. A rodent malaria, Plasmodium berghei, is experimentally transmitted to mice by merely probing of infective mosquito, Anopheles stephensi. Parasitol. Int. 51, 17-23 (2002).

5. Frischknecht, F. et al. Imaging movement of malaria parasites during transmission by Anopheles mosquitoes. Cell. Microbiol. 6, 687-694 (2004).
6. Natarajan, R. et al. Fluorescent Plasmodium berghei sporozoites and pre-erythrocytic stages: a new tool to study mosquito and mammalian host interactions with malaria parasites. Cell. Microbiol. 3, 371-379 (2001).

7. Franke-Fayard, B. et al. A Plasmodium berghei reference line that constitutively expresses GFP at a high level throughout the complete life cycle. Mol. Biochem. Parasitol. 137, 23-33 (2004).

8. Tarun, A.S. et al. Quantitative isolation and in vivo imaging of malaria parasite liver stages. Int. J. Parasitol. 36, 1283-1293 (2006).

9. Frevert, U. et al. Intravital observation of Plasmodium berghei sporozoite infection of the liver. PloS Biol. 6, e192 (2005).

10. Vanderberg, J.P. \& Frevert, U. Intravital microscopy demonstrating antibody-mediated immobilisation of Plasmodium berghei sporozoites injected into the skin by mosquitoes. Int. J. Parasitol. 9, 991-996 (2004).

11. Amino, R. et al. Quantitative imaging of Plasmodium transmission from mosquito to mammal. Nat. Med. 12, 220-224 (2006).

12. Sturm, A. et al. Manipulation of host hepatocytes by the malaria parasite for delivery into liver sinusoids. Science 313, 1287-1290 (2006).

13. Nakano, A. Spinning-disk confocal microscopy. A cutting-edge tool for imaging of membrane traffic. Cell Struct. Funct. 27, 349-355 (2002).

14. Sinden, R.E. et al. Maintenance of the Plasmodium berghei life cycle. Methods Mol. Med. 72, 25-40 (2002).

15. Fay, F.S., Carrington, W. \& Fogarty, K.E. Three-dimensional molecular distribution in single cells analysed using the digital imaging microscope. J. Microsc. 153 , 133-149 (1989).

16. Li, X., Sina, B. \& Rossignol, P.A. Probing behaviour and sporozoite delivery by Anopheles stephensi infected with Plasmodium berghei. Med. Vet. Entomol. 6, 57-61 (1992).

17. Medica, D.L. \& Sinnis, P. Quantitative dynamics of Plasmodium yoelii sporozoite transmission by infected anopheline mosquitoes. Infect. Immun. 73, 4363-4369 (2005). 